\section{EL PATRIMONIO CULTURAL COMO FACTOR DE DESARROLLO TURÍSTICO: ESTUDIO DE CASO EN LA CIUDAD DE CÓRDOBA}

\author{
Francisco González Santa Cruz \\ Universidad de Córdoba \\ ORCID iD: http://orcid.org/0000-0002-3872-7366 \\ francisco.gonzalez@uco.es \\ Tomás López-Guzmán \\ Universidad de Córdoba \\ ORCID iD: http://orcid.org/0000-0001-8800-8223 \\ tomas.lopez@uco.es
}

Cómo citar este artículo/Citation: González Santa Cruz, F. y López-Guzmán, T. (2017). El patrimonio cultural como factor de desarrollo turístico: estudio de caso en la ciudad de Córdoba. Arbor, 193 (786): a421. doi: http://dx.doi.org/10.3989/ arbor.2017.786n4009

Recibido: 4 abril 2016. Aceptado: 9 junio 2016.

RESUMEN: El patrimonio cultural inmaterial conforma la propia historia de los pueblos. El presente estudio trata de profundizar en el concepto de esta tipología de patrimonio y, específicamente, analiza la Fiesta de los Patios de Córdoba (España), declarada por la Organización de las Naciones Unidas para la Educación, la Ciencia y la Cultura (UNESCO) como Patrimonio Inmaterial de la Humanidad en 2012. El objetivo de este artículo es presentar los principales resultados de una investigación realizada a los turistas que participaron en la Fiesta de los Patios en 2014, desagregado por género. La investigación demuestra la presencia mayoritaria de turistas con estudios universitarios, con una edad superior a los 50 años y con un nivel de renta medio.

PALABRAS CLAVE: Patrimonio cultural; desarrollo turístico; género; Córdoba.

\section{CULTURAL HERITAGE AS A FACTOR OF TOURISM DEVELOPMENT: A CASE STUDY IN THE CITY OF CORDOBA}

Copyright: (C) 2017 CSIC. Este es un artículo de acceso abierto distribuido bajo los términos de la licencia Creative Commons Attribution (CC BY) España 3.0.

ABSTRACT: Intangible cultural heritage shapes the history of peoples. The present paper attempts to explore the concept of this type of heritage by focusing on the festivities of the Patios of Cordoba (Spain), declared by the United Nations Educational, Scientific and Cultural Organization (UNESCO) as an Intangible Culture Heritage in 2012. The aim of this paper is to present the main results of an investigation conducted on tourists participating in the Festival of the Patios in 2014, disaggregated by gender. The research shows that most of the tourists were university educated, with an average age above 50 years and an average income level.

KEYWORDS: Cultural heritage; tourism development; gender; Córdoba. 


\section{INTRODUCCIÓN}

En las últimas décadas, especialmente desde finales de los años ochenta del siglo XX, se han producido una serie de cambios sociales y económicos, entre los que destacan la liberación del comercio internacional, las mejoras y progresos en las tecnologías y las comunicaciones a nivel mundial, y la imposición, cada día más patente, de una globalización de carácter claramente mercantilista. Todos estos cambios están produciendo una serie de transformaciones en las sociedades hacia una mayor uniformidad en general y, específicamente, en sus formas de expresión social y festiva (Desantes Real, 2013). En el siglo XIX el folklore surge como una forma de afrontar la nueva situación económica y social derivada del cambio producido de manera general por la Revolución Industrial, que modificó todos los valores culturales predominantes hasta la fecha (Velasco Maillo, 1990). En el mismo sentido, en la actualidad la preocupación por el patrimonio inmaterial es entendida como una reacción similar, pero en este caso ante las condiciones de vida generalizadas por el proceso de globalización. Ante la supuesta homogeneización cultural que conlleva la misma, se impone la necesidad de reivindicar lo propio y construir patrimonios culturales que nos diferencien (Gómez Pellón, 2002).

En este sentido la salvaguardia de los valores culturales diferenciadores se ha convertido en una necesidad para el mantenimiento de aquellas tradiciones que nos han conformado como pueblos distintos, ricos en costumbres y expresiones populares, concretadas en el patrimonio material e inmaterial que les confiere permanencia en el tiempo, superando la máxima del individualismo en las sociedades avanzadas y volviendo a dar valor a lo comunitario. Con esta idea, la UNESCO (Organización de las Naciones Unidas para la Educación, la Ciencia y la Cultura), en la reunión realizada en París en el año 2001, intentó establecer una definición provisional del Patrimonio Cultural Inmaterial (UNESCO, 2001), entendiendo que lo inmaterial nos acerca a la noción de identidad, comprendiendo dentro de este nuevo concepto (Castelli González, 2006) los procesos aprendidos por los pueblos junto con el saber, las destrezas y la creatividad que los definen y son creadoas por ellos, los productos que elaboran, y los recursos, espacios y otros aspectos del contexto social y natural necesarios para su sostenibilidad.

Estas expresiones culturales mantienen su valor mientras permanecen vivas en las comunidades que las fueron generando. Podemos señalar, por tanto, que se ha producido un cambio de paradigma en la comunidad internacional, que desde hace poco tiempo está preocupada por el rescate de lo que podemos considerar inmaterial, dado el contenido que tiene de nuestro propio pasado, y cuya necesidad de conservación y salvaguardia está al mismo nivel que los bienes materiales de interés cultural (Quiros Vicente y Casas Pérez, 2011; Quintero Rivera, 2002). En todo caso, no debemos ver el patrimonio intangible únicamente como un recurso al que salvaguardar por el interés económico que tiene en el mismo, sino que su significación más importante es sobresalir como factor de identidad y sociabilidad, dado su gran simbolismo y potestad de creación identitaria (Olivera, 2011), otorgando por tanto a dicho valor un importante privilegio de salvaguardia. En este sentido, la preocupación por la sostenibilidad del patrimonio nos determina como sociedades de futuro, al tiempo conservadora de sus requisitos tradicionales y abierta a los cambios derivados de la contemporaneidad (Plasencia Martín, 2007).

Sin embargo, la realidad económica que nos rodea hace necesario también el uso de variables de segmentación de los visitantes, por su potencial utilización en la adopción de políticas públicas y privadas encaminadas a la mejora de la satisfacción de estos, siendo imprescindible para ello la identificación de los perfiles de los visitantes actuales y, por ende, de los potenciales (Fernández-Huerga, 2010; Sánchez Sánchez y Fernández Puente, 2014; Wedel y Kamakura, 2000; Kotler, Armstrong, Saunders y Wong, 1999). Así en experiencias comparadas dentro de otros ámbitos como, por ejemplo, en el turismo del vino se han realizado multitud de estudios para determinar los perfiles sociodemográficos de los turistas, siendo estudiados factores como la edad, la ocupación, los niveles educativos, los ingresos o la renta disponible para así poder adaptar mejor la oferta a quienes buscan esta tipología de visitantes (entre otros, Tassiopoulos, Nuntsu y Haydam, 2004; Bruwer, 2004; Jolley, 2002; Howley y Van Westering, 2000; Dodd y Gustafson, 1997).

El objetivo fundamental que persigue el presente artículo es, de manera general, mejorar el conocimiento sobre el patrimonio inmaterial de la humanidad y, específicamente, de la Fiesta de los Patios de Córdoba y, dentro de este análisis, conocer el perfil sociodemográfico de los visitantes de la Fiesta, determinando, del mismo modo, la influencia que tiene el género de estos en las diferentes facetas que han sido objeto de estudio en esta investigación. Con estos avances también se pretende aportar a las entidades públicas y privadas que intervienen en la promoción, 
organización y desarrollo de la Fiesta de los Patios de una serie de parámetros que les permitan la mejora de la percepción que los visitantes actuales tienen de ella (a través de la orientación clara de sus políticas y actuaciones). A efectos de dar una mayor claridad expositiva, la estructura del presente artículo comienza, tras esta introducción, con una revisión teórica que fundamenta el concepto objeto de estudio, continuando con una breve descripción del área geográfica donde se ha desarrollado el trabajo de campo, la metodología empleada y los resultados derivados de la investigación, que persiguen el avance del conocimiento científico en la materia. Para finalizar se presentan las conclusiones más destacadas, la principal limitación de la investigación, el planteamiento de las futuras líneas de investigación que se consideran más adecuadas y las referencias bibliográficas utilizadas.

\section{REVISIÓN DE LA BIBLIOGRAFÍA}

\section{a. Conceptualización general}

El concepto de patrimonio cultural, como cualquier construcción social, es dinámico y refleja el avance que producen los cambios sociales, políticos y económicos de las sociedades que lo producen o definen (Carrera Díaz y Delgado Méndez, 2012). Existen muchas manifestaciones del patrimonio cultural que no tienen apariencia física, y este patrimonio cultural "especial" viene integrado por "conocimientos tradicionales" y por "expresiones culturales tradicionales y populares" directamente atribuibles a "comunidades" de muy diversa índole que pueden tener su origen en consideraciones derivadas del indigenismo, de la etnia, de la ruralidad, de la localidad o simplemente del hecho de compartir determinados valores culturales o sociales. Como no tienen entidad física, suelen denominarse "patrimonio inmaterial" (Desantes Real, 2013). En cualquier caso, la mayoría de las expresiones culturales inmateriales tienen reflejo en el mundo material, es decir, en cierto modo, lo material y lo inmaterial son inseparables (Ruggles y Silverman, 2009).

Pero cuando hablamos de patrimonio inmaterial nos podemos estar refiriendo a multitud de expresiones populares. Olivera (2011) concretó esta tipología de patrimonio ciñéndolo a las actividades y saberes que se manifiestan, principalmente, en fiestas populares; rituales, creencias y mitos; manifestaciones escénicas y espectáculos tradicionales; conocimientos y usos relacionados con la naturaleza y el universo (medicina y farmacopea tradicional, cosmologías); tradiciones culinarias y fiestas gastronómicas; habilidades artesanas y tesoros humanos vivos; formas de expresión musical, canto y danzas populares; manifestaciones lúdicas y deportes tradicionales; lenguas, tradiciones y expresiones orales, epopeyas heroicas y leyendas; usos y costumbres sociales; espacios culturales con alta presencia inmaterial; hitos de la memoria colectiva (lugares de encuentro); especiales formas de adaptación al medio natural; espacios imaginarios y de inspiración de obras pictóricas, musicales o literarias; etc.

Durante las últimas décadas, gracias a la expansión realizada por el constructo "patrimonio" (Santamarina Campos, 2013; Ariño Villarroya, 2002), hemos asistido al reconocimiento global y globalizado de otros patrimonios potenciales que, hasta este momento, eran o bien no reconocidos como tales o bien minusvalorados como patrimonios "menores". Del mismo modo, se está produciendo un cambio de enfoque dentro del campo de las Ciencias Sociales mostrando un interés especial por los procesos en detrimento de los objetos, lo que ha llevado al nacimiento de una nueva definición de patrimonio como la entidad compuesta por expresiones que se manifiestan de manera compleja y diversa a través de las costumbres sociales (Sánchez Carretero, 2005; Bouchenaki, 2004). Esta nueva perspectiva precisó de la identificación de las costumbres y de los sistemas de creencias para hacer que el enfoque patrimonial fuera aplicable a los aspectos tanto materiales como inmateriales (Mariano, 2011). Desde este nuevo enfoque, el patrimonio cultural inmaterial se revaloriza como fuente de diversidad, identidad, creatividad, y, además, como práctica y conocimiento de quienes lo portan (Bouchenaki, 2004; Kirshenblatt-Gimblet, 2004). En este contexto, se entiende al patrimonio como construcción social (Hernando Gonzalo, 2009; Mantecón, 1998), sustentado en la propia riqueza de la sociedad que lo conforma. Así el patrimonio cultural se ha ido conformando como un ámbito interdisciplinar que se acerca a las realidades socioculturales y los colectivos que, de una u otra forma, le dan vida (Delgado Méndez, 2008).

En cualquier caso, existen numerosos autores (entre otros, Villaseñor Alonso y Zolla Márquez, 2012; Giménez, 2007) que señalan que hablar de un patrimonio material en contraposición con un patrimonio inmaterial representa una dicotomía conceptual innecesaria, ya que se está refiriendo a dos caras de una misma "moneda". Del mismo modo, desde los años ochenta del pasado siglo, el patrimonio inmaterial ha sido objeto de un intenso proceso de institucionalización y construcción jurídica (Cejudo, 2014; Di Giovine, 2009; Bouchenaki y Kurin, 1989). No obstante, antes de su aparición ya se utilizaban las categorías "riquezas ar- 
tísticas" o "tesoros artísticos" para referirse al legado espiritual de una nación o a su patrimonio económico (Boude Figueredo y Luna, 2013).

Actualmente nos resulta bastante normalizada la noción de patrimonio inmaterial, pero su conceptualización como la conocemos hoy en día es relativamente reciente. En las últimas décadas del siglo pasado, se realizaron muchos intentos por parte de la UNESCO por buscar un concepto fijo y uniforme de lo que podría comprender el patrimonio inmaterial, pero no es hasta el año 2002 en la Reunión Internacional de Expertos de la UNESCO en Río de Janeiro donde se recalcó la relevancia de adoptar un concepto flexible de salvaguarda del patrimonio cultural inmaterial (Villaseñor Alonso y Zolla Márquez, 2012). En esta reunión se enfatizó que los miembros de las comunidades practicantes son quienes deben decidir sobre las prácticas culturales que deberán ser salvaguardadas, así como sobre las formas en que estas deben ser protegidas. Asimismo, se expresó la inquietud de que personas externas se apropiaran de los recursos culturales y surgió la preocupación sobre la autoridad en la proclamación del patrimonio cultural inmaterial (Arizpe, 2009).

Partiendo de estos fundamentos, la convención del 17 de octubre de 2003 en París, bajo el auspicio de la UNESCO, para la salvaguardia del patrimonio cultural inmaterial, concluye que:

Se entiende por patrimonio cultural inmaterial -- los usos, representaciones, expresiones, conocimientos y técnicas -junto con los instrumentos, objetos, artefactos y espacios culturales que les son inherentes- que las comunidades, los grupos y en algunos casos los individuos reconozcan como parte integrante de su patrimonio cultural. Este patrimonio cultural inmaterial, que se transmite de generación en generación, es recreado constantemente por las comunidades y grupos en función de su entorno, su interacción con la naturaleza y su historia, infundiéndoles un sentimiento de identidad y continuidad y contribuyendo así a promover el respeto de la diversidad cultural y la creatividad humana (UNESCO, 2003).

De la definición anterior es posible sacar algunas notas definitorias del patrimonio inmaterial como es su carácter intangible, transformable, adaptable y dinámico; está en constante construcción, evoluciona o involuciona, y necesita ser recreado constantemente para preservarse (Boude Figueredo y Luna, 2013). No obstante, la amplitud de manifestaciones culturales que tienen cabida bajo esta definición es de tal magnitud que ha provocado, como señalan Ruggles y Silver- man (2009), que cualquier publicación sobre patrimonio inmaterial tenga que dedicar un amplio espacio a la cuestión de la delimitación del concepto. En este sentido, según López López (2011, p.1.895), hay “tres aspectos que, en la práctica, siempre están presentes en la transformación patrimonial de determinadas manifestaciones culturales: su vinculación con la idea de tradición, su aparente carácter desconflictualizado y su capacidad para expresar cierto tipo de valores colectivos caracterizados de una forma más o menos abstracta" (p. 1895).

\section{b. La Fiesta como parte del concepto inmaterial de cultura}

El conocimiento de la cultura por parte de los grupos regionales es un buen reflejo de cómo el saber tradicional garantiza la conservación del patrimonio tanto material como inmaterial. Esta diversidad nos ayuda a entender no sólo nuestra cultura sino otras, de manera que la herencia que nos lega la ciencia de la conservación contribuye al fortalecimiento de las identidades locales y regionales (Aguilar Solves, 2014). Por ello, es importante tener presente que los motivos para la Fiesta no los suministra la historia que revive, sino la propia representación de la misma en la actualidad. En este sentido, el estudio de las fiestas y su relación con los procesos de identificación colectiva ha sido una de las temáticas más recurrentes de los estudios científicos de las últimas décadas tanto en España como en Andalucía (Brisset, 1992). Pero, como advierte Prat i Carós (1999), a menudo encontramos que las nociones de folklore, cultura popular y patrimonio cultural hacen referencia a unos mismos objetos de estudio (García-Olivares, 1997). Y uno de esos referentes, uno de esos objetos, ha sido la Fiesta.

Partiendo de esta visión, y siguiendo el estudio realizado por López López y Ruiz Ruiz (2013), podríamos señalar que la Fiesta de los Patios de Córdoba tiene un importante carácter patrimonial, al cumplir con las tres dimensiones que estos autores (en su estudio sobre la Fiesta de los Mosqueteros del Santísimo Sacramento de Béznar en Granada) consideran necesarias en este ámbito: 1) raigambre histórica de la celebración y sus implicaciones en la memoria colectiva, 2) centralidad del fenómeno dentro de los procesos de identificación local, y 3) diversas formas de sociabilidad que propicia el contexto festivo.

A efectos de determinar la posible existencia de los puntos anteriores en la Fiesta de los Patios de Córdoba, debemos señalar que la propia esencia de ésta se enclava en la conceptualización de celebración con 
raigambre histórica. En este sentido, el origen de esta tipología de casa en Córdoba se asienta a finales del siglo XIX y principios del siglo XX para dar respuesta a las necesidades de alojamiento que precisaban las personas que se trasladaban a la ciudad desde entornos rurales (véase Aspectos culturales, sociales, festivos, económicos y de vida cotidiana asociados al patio tradicional cordobés). Por su parte, la significación de la celebración en la llegada de la primavera con todo su carácter de alegría (flores, macetas, etc.) y la apertura al vecindario de los patios, da un valor patrimonial de identificación local muy importante a la celebración en sí, ya que es en los patios donde los vecinos, junto con sus familias y amigos, se reúnen y convierten a estos en lugares de esparcimiento y de celebración colectiva. $Y$ finalmente, en el aspecto de la sociabilidad y formas de participación, la propia UNESCO señala que la Fiesta de los Patios de Córdoba promueve la función del patio como lugar de encuentro intercultural entre las personas, y fomenta un modo de vida colectivo sostenible, basado en el establecimiento de vínculos sólidos y de redes de solidaridad e intercambios entre vecinos, estimulando al mismo tiempo la adquisición de conocimientos y el respecto por la naturaleza.

\section{c. Relación del turismo con el patrimonio inmaterial}

El turismo tiene una relación directa con el disfrute de los activos del patrimonio inmaterial en el marco de las experiencias del turismo cultural. Podemos considerar al turismo cultural como aquel cuya motivación es conocer, comprender y disfrutar de elementos y rasgos distintivos, espirituales, materiales e intelectuales, que caracteriza a una comunidad o grupo social concreto. Dentro del turismo actual, aquel que tiene su fundamento en la cultura, se ha convertido en uno de los fenómenos más importantes y diversos de nuestro tiempo, abierto a un continuo proceso de innovación, mejora y creación de nuevos productos en respuesta a las nuevas demandas de experiencias turísticas diversificadas (Brunet y Alarcón, 2008) y, sin duda, a la evolución del conocimiento en materia de gestión cultural (véase Turismo y patrimonio cultural inmaterial). En este sentido, los intereses del sector turístico deben reconocer los conceptos, ideales y prácticas inherentes a la gestión del patrimonio cultural, pero, de manera recíproca, aquellos que gestionan los patrimonios culturales deben entender qué es y cómo funciona el turismo. De esta manera, el ámbito turístico y el patrimonial cultural podrán asociarse buscando la simbiosis en sus intercambios.

En cualquier caso, la relación entre patrimonio y turismo, aunque parezca algo muy cercano en el tiem- po y perteneciente, principalmente, al siglo XXI, no es tan actual, ya que desde finales del siglo pasado, el patrimonio cultural ha sido atractivo para el turismo (Troncoso y Almirón, 2005, Alonso González y Macías Vázquez, 2014). Pero es innegable que en los últimos años se ha acrecentado de manera destacada dicha relación, más quizás por su valor económico que cultural (Leijonhufvud, 2011; Quintana y Stagno, 2009). Así los agentes turísticos y las autoridades (locales, regionales, nacionales e internacionales) muestran cada vez mayor interés en concebir productos turísticos asociados con el patrimonio cultural inmaterial que, a su vez, contribuyan a su salvaguardia, reforzando la diversidad cultural de los destinos, enriqueciendo su oferta turística. En todo caso, los activos patrimoniales no tienen un carácter eminentemente comercial, sino que son de dominio público, por lo que la gestión, promoción y salvaguardia están entre las competencias básicas de las diferentes administraciones públicas (Cuasapás Ponce, 2013). Este crecimiento del turismo vinculado al patrimonio está relacionado, por tanto, con la aparición de una nueva tipología de turista, aquel que está preocupado por acercarse y conocer la naturaleza y las culturas, que busca consumir nuevos productos turísticos diferentes a los habituales (Troncoso y Almirón, 2005).

\section{ÁMBITO GEOGRÁFICO}

La ciudad de Córdoba cuenta con una población total de 328.704 habitantes (Instituto Nacional de Estadística, 2014a), de los que el 48,06\% son hombres y el $51,94 \%$ mujeres. Es la tercera ciudad por tamaño y población de la región de Andalucía, contando con una extensión municipal de $1.245 \mathrm{~km}^{2}$. Su casco histórico (Judería) fue declarado Patrimonio Material de la Humanidad por la UNESCO en 1994. Diez años antes, en 1984, lo había sido la Mezquita-Catedral de Córdoba.

La Fiesta de los Patios se celebra durante el mes de mayo en Córdoba. En dicha festividad, los vecinos de las casas típicas, cuya arquitectura bebe directamente de la fisonomía de las viviendas romanas, y que luego adaptaron los árabes, y que pasan todo el año cuidando de las plantas, abren sus casas a los visitantes para que puedan visitarlas y, sobre todo, participar en la Fiesta de los Patios. Esos días, los inquilinos ofrecen su hospitalidad a los foráneos y a la propia comunidad local. Esta tipología de casas se va adaptando a la situación socioeconómica de la ciudad de Córdoba y a los usos sociales imperantes en cada época histórica (véase Aspectos culturales, sociales, festivos, económicos y de vida cotidiana asociados al patio tradicional cordobés). 
El año 2013 fue el primero en el que la Fiesta de los Patios de Córdoba se celebró ya con la declaración de Patrimonio Inmaterial de la Humanidad, lo cual supuso un fuerte incremento en el número de visitantes. Así, los ingresos estimados en el citado año 2013 y derivados de la Fiesta de los Patios, también aumentaronó por encima del 50\%, superando los 6 millones de euros (véase Estudio sobre la repercusión económica en la ciudad de Córdoba de la Fiesta de los Patios en mayo de 2013). Hemos de resaltar que la entrada a los patios por parte de los visitantes es totalmente gratuita y que el número de patios abiertos al público en la edición de 2014 fue de 65, agrupados en seis itinerarios que se distribuían por diferentes zonas de la ciudad.

\section{METODOLOGÍA}

Para llevar a cabo la investigación se realizó un trabajo de campo entre los días 5 y 18 de mayo de 2014, coincidiendo con las fechas de celebración de la Fiesta de los Patios en Córdoba, con el que se perseguía conocer, entre otras cuestiones, el perfil sociodemográfico de los visitantes y, específicamente, determinar la influencia que el género podría tener en los distintos ítems consultados. El cuestionario, desde donde se obtuvieron los resultados que serán objeto de análisis y que se presentan en el apartado siguiente, fueron cumplimentados por los turistas que cumplían dos condiciones de manera simultánea: primera, que se encontrasen visitando cualquiera de los patios definidos dentro de los itinerarios oficiales; $y$, segunda, que hubiesen visitado, al menos, dos patios.

El cuestionario se basa en trabajos previos, sobre temáticas y situaciones similares (Lourenço-Gomes, Costa Pinto y Rebelo, 2014; Chen y Chen 2010; Nicholas y Thapa, 2010). El mismo día que comenzó la Fiesta de los Patios se realizó un pre-test de 30 cuestionarios para detectar posibles desviaciones y errores, aplicando a la versión definitiva las mejoras detectadas en el mismo. En el cuestionario se establecían ítems que mezclaban preguntas sobre la valoración de la opinión del visitante (mediante una escala de Likert de cinco puntos), respuestas sí/no, y cuestiones tanto cerradas como abiertas. En la cumplimentación de los cuestionarios, que eran totalmente anónimos, estaban presentes los encuestadores para resolver las dudas que pudieran surgir. Los cuestionarios se distribuyeron en cuatro idiomas (español, inglés, francés y alemán).

En el trabajo de campo desarrollado se realizaron 992 encuestas, de las cuales 960 fueron válidas. El número total de personas que visitaron los Patios en
2013, según fuentes del Ayuntamiento de Córdoba, fue de unas 80.000 personas, de las cuales aproximadamente un $52 \%$ eran turistas. Por tanto, hemos considerado como población objetivo 41.600 personas. Con esta población objetivo, el error muestral de la investigación fue del +/- 3,13\%. El índice de fiabilidad según el Alfa de Cronbach fue de 0,782, lo cual da sustento de validez al trabajo de campo (Nunnally y Bernstein, 1994).

En la tabla 1 se presenta la ficha técnica de la investigación realizada en la Fiesta de los Patios de Córdoba en 2014.

Del mismo modo, se realizó una estratificación de los turistas por países y, en concreto en España, por regiones, siguiendo el patrón porcentual definido en la Encuesta de Ocupación Hotelera correspondiente a mayo de 2012 (último dato disponible de carácter mensual en el momento de la realización del trabajo de campo) en la ciudad de Córdoba (Instituto Nacional de Estadística, 2014b) y de acuerdo cona los datos del Observatorio Turístico de Córdoba. Informe 2013. Con los datos extraídos de las citadas fuentes, podemos determinar que el 53,52 \% de los turistas alojados en los establecimientos hoteleros de la ciudad de Córdoba en mayo de 2012 fueron españoles, y, el restante, $46,48 \%$, extranjeros. En la tabla 2 se presentan los porcentajes por comunidades autónomas procedentes a partir de los datos del Observatorio Turístico de Córdoba de 2012, y el número de cuestionarios realizados (y su porcentaje) en esta investigación. En la tabla 3 se presentan los datos porcentuales por países extranjeros según la Encuesta de Ocupación Hotelera (Instituto Nacional de Estadística, 2014b) y los cuestionarios y porcentajes correspondientes alcanzados en el trabajo de campo. Una vez definidos los porcentajes de turistas que se iban a encuestar de acuerdo con su nacionalidad o región de procedencia, se utilizó un muestreo de conveniencia, comúnmente utilizado por la doctrina científica en este tipo de investigaciones, donde los encuestados están disponibles para ser preguntados en un espacio y tiempo determinado (Finn, Walton y Elliot-White, 2000).

Como se puede apreciar en las tablas 2 y 3 , la similitud entre los datos porcentuales estratificados de las diferentes fuentes estadísticas oficiales (Observatorio Turístico de Córdoba. Informe 2013 y Encuesta de Ocupación Hotelera, respectivamente) y la distribución porcentual conseguida en el trabajo de campo son prácticamente idénticas, por lo que también con este dato podemos determinar la fiabilidad de la investigación de campo realizada. 
Tabla 1. Ficha técnica de la investigación sobre la demanda

\begin{tabular}{|l|l|}
\hline $\begin{array}{l}\text { Número de personas que visitan los Patios } \\
\text { (referenciado al año 2013) }\end{array}$ & 80.000 \\
\hline Muestra & 960 cuestionarios \\
\hline Error muestral & $+/-3,13 \%$ \\
\hline Procedimiento & Muestreo aleatorio estratificado \\
\hline Periodo de realización & Mayo de 2014 \\
\hline Control de la muestra & $\begin{array}{l}\text { Realización y supervisión del trabajo de campo por los autores de } \\
\text { la investigación }\end{array}$ \\
\hline
\end{tabular}

Fuente: elaboración propia.

Tabla 2. Distribución porcentual por comunidades autónomas

\begin{tabular}{|l|c|c|}
\hline Comunidad autónoma & $\begin{array}{c}\text { Observatorio Turístico de Córdoba } \\
\mathbf{2 0 1 2}\end{array}$ & \begin{tabular}{c} 
Cuestionarios realizados (N = 504) \\
\hline Andalucía
\end{tabular} 43,60\% \\
\hline Madrid & $21,83 \%$ & $\mathbf{4 1 , 8 \%}$ \\
\hline Cataluña & $7,62 \%$ & $8,0 \%$ \\
\hline Comunidad Valenciana & $5,19 \%$ & $6,0 \%$ \\
\hline Castilla-La Mancha & $4,10 \%$ & $4,2 \%$ \\
\hline Castilla y León & $2,92 \%$ & $3,4 \%$ \\
\hline Extremadura & $2,72 \%$ & $3,3 \%$ \\
\hline Resto de España & $12,12 \%$ & $12,7 \%$ \\
\hline
\end{tabular}

Fuente: elaboración propia a partir de Observatorio Turístico Córdoba. Informe 2013.

Tabla 3. Distribución porcentual por países extranjeros

\begin{tabular}{|l|c|c|}
\hline Países & Encuesta de Ocupación Hotelera & Encuestas realizadas (N = 456) \\
\hline Francia & $20,23 \%$ & $20,4 \%$ \\
\hline Alemania & $12,85 \%$ & $12,9 \%$ \\
\hline Estados Unidos & $9,34 \%$ & $9,2 \%$ \\
\hline Reino Unido & $8,82 \%$ & $11,6 \%$ \\
\hline Italia & $6,15 \%$ & $7,7 \%$ \\
\hline Países Bajos & $5,19 \%$ & $5,5 \%$ \\
\hline Japón & $4,93 \%$ & $3,7 \%$ \\
\hline Bélgica & $3,14 \%$ & $2,9 \%$ \\
\hline Portugal & $1,39 \%$ & $2,4 \%$ \\
\hline Otros & $27,9 \%$ & $23,7 \%$ \\
\hline
\end{tabular}

Fuente: elaboración propia a partir de Instituto Nacional de Estadística (2014).

Para el análisis general de las variables se han empleado los métodos descriptivos básicos para las variables cualitativas, obteniendo el número de casos presentes en cada categoría y el porcentaje correspondiente, y para las variables cuantitativas hemos obtenido la media y la desviación típica. En las ta- 
blas de contingencia obtenemos la frecuencia y el porcentaje de casos que presentan las dos características de forma conjunta. Para conocer si entre las dos variables hay o no dependencia hemos realizado la prueba Chi-cuadrado. Se comprueba también si las proporciones de columna son o no diferentes. La comparación de medias entre dos grupos se ha realizado mediante el test de t-Student bajo el supuesto de normalidad comprobada con el test de Kolmogorov-Smirnov. El análisis estadístico se realizó con el programa SPSS 21.0 para Windows. Las diferencias consideradas estadísticamente significativas son aquellas cuya $p<0.05$.

\section{RESULTADOS DE LA INVESTIGACIÓN Y DISCUSIÓN}

Uno de los objetivos de esta investigación es determinar el perfil sociodemográficos de los visitantes a la Fiesta de los Patios de Córdoba, diferenciado por género. Así, en la tabla 4 se presentan los principales resultados, diferenciados por género, en relación con la edad, nivel educativo, país de procedencia y renta mensual de los turistas que han visitado el citado patrimonio.
Como se puede apreciar de la tabla 4, la primera cuestión que hay quea destacar es que del total de encuestados, el $46,5 \%$ eran hombres y el $53,5 \%$ restante mujeres. Por tanto, existe un pequeño desequilibrio pero que permite aseverar una casi paridad entre los encuestados. En líneas generales se pueden extraer algunas conclusiones interesantes, para algunas de las variables independientes analizadas:

a) Edad: el 50,8\% de los visitantes varones tiene más de 50 años y, en cambio, las mujeres con el citado rango de edad, se quedan en el $43,8 \%$. Además, en el caso de las mujeres, casi otro $40 \%$ (concretamente el 39,4\%) tiene menos de 40 años. Por tanto, la primera conclusión que podríamos sacar de estos datos es la necesidad de fomentar la promoción, especialmente en el caso de los hombres, en el segmento de edad inferior a 39 años, ya que también de esta forma se podrían tener un relevo generacional con el paso del tiempo en los propios visitantes. En las mujeres este relevo parece estar más asegurado.

Tabla 4. Perfil sociodemográfico de los turistas

\begin{tabular}{|c|c|c|c|}
\hline \multicolumn{2}{|l|}{ Variables } & \multirow{2}{*}{$\begin{array}{c}\text { Hombres }(\mathbf{N}=\mathbf{4 4 5}) \\
13,7 \%\end{array}$} & \multirow{2}{*}{$\begin{array}{c}\text { Mujeres ( } \mathbf{N}=\mathbf{5 1 2} \text { ) } \\
19,4 \%\end{array}$} \\
\hline \multirow{5}{*}{$\begin{array}{l}\text { Edad } \\
(\mathrm{N}=956)\end{array}$} & Menos de 30 años & & \\
\hline & 30-39 años & $18,0 \%$ & $20,0 \%$ \\
\hline & 40-49 años & $17,5 \%$ & $16,6 \%$ \\
\hline & 50-59 años & $22,5 \%$ & $21,9 \%$ \\
\hline & 60 o más años & $28,3 \%$ & $21,9 \%$ \\
\hline \multirow{3}{*}{$\begin{array}{l}\text { Nivel Educativo } \\
(\mathrm{N}=942)\end{array}$} & Primaria & $3,4 \%$ & $6,0 \%$ \\
\hline & Secundaria & $24,1 \%$ & $22,9 \%$ \\
\hline & Universitaria & $72,2 \%$ & $71,0 \%$ \\
\hline \multirow{6}{*}{$\begin{array}{l}\text { País } \\
\text { (N =957) }\end{array}$} & España & $51,7 \%$ & $53,3 \%$ \\
\hline & Reino Unido & $6,1 \%$ & $4,9 \%$ \\
\hline & Francia & $11,7 \%$ & $8,0 \%$ \\
\hline & Alemania & $7,2 \%$ & $5,3 \%$ \\
\hline & Italia & $3,1 \%$ & $4,1 \%$ \\
\hline & Otros & $20,2 \%$ & $24,4 \%$ \\
\hline \multirow{5}{*}{$\begin{array}{l}\text { Renta mensual } \\
(\mathrm{N}=826)\end{array}$} & Menos de 700 euros /mes & $5,2 \%$ & $9,5 \%$ \\
\hline & De 700 a 1.000 euros & $6,5 \%$ & $11,6 \%$ \\
\hline & De 1.001 a 1.500 euros & $15,6 \%$ & $22,7 \%$ \\
\hline & De 1.501 a 2.500 euros & $30,4 \%$ & $28,2 \%$ \\
\hline & Más de 2.500 euros & $42,3 \%$ & 28,05 \\
\hline
\end{tabular}

Fuente: elaboración propia 
b) Nivel educativo: con gran similitud en ambos sexos (como se podía intuir de la falta de significación estadística del coeficiente de contingencia), la gran mayoría de los visitantes de los patios tiene estudios universitarios (más de 70\% en los dos casos). Este resultado podría ser considerado dentro de la normalidad, ya que existe una relación bastante intensa entre los niveles educativos superiores y el turismo cultural.

c) País: como se ve en la tabla 4, la mayor presencia de visitantes es de españoles (muy similar en ambos sexos), estando algo por encima del 50\%, y que responde a la metodología utilizada en esta investigación en cuanto al muestreo estratificado por países. No obstante, en cuanto a la distribución de los visitantes extranjeros, cabe señalar la diversidad de orígenes de éstos, determinando este aspecto el amplio conocimiento que de la Fiesta de los Patios de Córdoba se tienen en muchos países de nuestro entorno y lejos de él. De hecho, en esta investigación se recogen perfiles de personas encuestadas de 39 países.

d) Renta mensual: finalmente hay que destacar que más del $40 \%$ de los hombres que visitan la Fiesta de los Patios, tiene un salario mensual neto superior a 2.500 euros al mes, cuando en el caso de las mujeres dicha cuantía no llega al 30\%, lo cual de- terminan la tradicional brecha salarial por razón de género. Sin embargo, esta cuestión no está relacionada con el nivel de estudios, como se ha señalado en el apartado b) anterior.

En definitiva, viendo los datos anteriores, los poderes públicos y organizaciones empresariales que participan en el desarrollo y expansión turística de la Fiesta de los Patios, podrían poner en marcha medidas de promoción orientada a perfiles masculinos menores de 39 años dentro del ámbito universitario o egresados, ya que de esta forma fomentarían la participación de perfiles, actualmente, infrarrepresentados en la participación en la Fiesta y, como ha quedado reflejado, con un poder adquisitivo bastante destacado. $\mathrm{Si}$ a esta orientación le añadimos la promoción internacional en dicho ámbito, diversificarían además el espectro de potenciales mercados emisores de turistas extranjeros que participen en la celebración de este Patrimonio Inmaterial de la Humanidad.

Por otra parte, a efectos de determinar la importancia relativa de la Fiesta de los Patios de Córdoba, como motivación de visita a la ciudad, sobre los otros atractivos turísticos de la misma, y todo ello segmentado por sexo, se planteó a los turistas que valorasen una serie de motivos en una Escala de Likert de 5 puntos (tabla 5).

Tabla 5. Motivación para visitar distintos atractivos de la ciudad de Córdoba. Comparación entre hombres y mujeres

\begin{tabular}{|c|c|c|c|c|c|c|}
\hline \multirow{3}{*}{ Variables } & \multicolumn{2}{|c|}{ Sexo } & \multicolumn{4}{|c|}{ Prueba T para la igualdad de medias ${ }^{\dagger}$} \\
\hline & Hombre & Mujer & \multirow{2}{*}{ Dif. medias } & \multirow{2}{*}{ ET dif. } & \multirow{2}{*}{$t_{881}$} & \multirow{2}{*}{$\mathbf{p}$} \\
\hline & Media (DT) & Media (DT) & & & & \\
\hline Visitar los Patios & $3,81(1,3)$ & $4,16(2,2)$ & $-0,34$ & 0,12 & $-2,76$ & $0,006 * *$ \\
\hline Degustar la gastronomía cordobesa & $3,41(1,3)$ & $3,5(1,3)$ & $-0,09$ & 0,09 & $-0,98$ & 0,329 \\
\hline $\begin{array}{l}\text { Conocer Patrimonio Material de } \\
\text { Córdoba }\end{array}$ & $4,3(1)$ & $4,31(1)$ & $-0,01$ & 0,07 & $-0,15$ & 0,879 \\
\hline $\begin{array}{l}\text { Conocer algunos museos de la } \\
\text { ciudad }\end{array}$ & $2,88(1,4)$ & $2,84(1,4)$ & 0,04 & 0,10 & 0,42 & 0,676 \\
\hline Entretenimiento & $3(1,3)$ & $3,18(1,4)$ & $-0,18$ & 0,10 & $-1,70$ & 0,090 \\
\hline Relajación & $3,14(1,3)$ & $3,15(1,4)$ & $-0,01$ & 0,11 & $-0,12$ & 0,905 \\
\hline Pasar un día fuera & $3,19(1,5)$ & $3,33(1,5)$ & $-0,14$ & 0,12 & $-1,22$ & 0,224 \\
\hline Conocer la ciudad & $4,13(1,1)$ & $4,09(1,3)$ & 0,04 & 0,08 & 0,43 & 0,669 \\
\hline Visitar a amigos y familiares & $2,01(1,6)$ & $2,34(1,8)$ & $-0,33$ & 0,13 & $-2,46$ & $0,014^{*}$ \\
\hline Motivos de trabajo & $1,36(1)$ & $1,54(1,3)$ & $-0,19$ & 0,10 & $-1,92$ & $0,045^{*}$ \\
\hline
\end{tabular}

† Homogeneidad asumida. ${ }^{*} \mathrm{p}<0.05 * * \mathrm{p}<0.01$ 
De acuerdo con la tabla 5 anterior, observamos que los aspectos que motivaron la visita a Córdoba y que presentan diferencias significativas entre hombres y mujeres fueron: "Fiesta de los Patios", "Visitar amigos y familiares" y "Por motivos laborales", siendo las mujeres las que presentan mayor motivación en estos aspectos que los hombres $(p<0.05)$. Por su parte, en los aspectos descriptivos, vemos que en ambos sexos el principal atractivo turístico que motivó la visita a Córdoba, en el periodo de la realización de la investigación, fue conocer el patrimonio material de la ciudad (fundamentalmente, y como se ha señalado anteriormente, el casco histórico y/o la Mezquita-Catedral), pero le sigue en importancia, para las mujeres, como motivación la visita a la Fiesta de los Patios (4,16 puntos), cuando para los hombres se sitúa en la tercera posición en motivación (3,81 puntos). Siguiendo con el análisis desagregado, en el lado contrario en ambos sexos, el menor valor motivacional lo tienen las cuestiones relacionadas con el trabajo, siendo algo mayor en las mujeres (1,54 puntos) que en los hombres (1,36 puntos). En todo caso resulta significativo que el conocimiento de los diferentes museos de la ciudad reciba una calificación baja en la motivación para visitar la misma, por parte de los turistas encuestados tanto hombres $(2,88)$ como mujeres (2,84). Esta cuestión debería ser objeto de respuesta por las autoridades locales competentes desarrollando, por ejemplo, el concepto denominado ecomuseo (Gilabert González, 2015), que se trata de una institución basada en la idea de conservar y presentar in situ los elementos patrimoniales de un territorio y donde la comunidad local es una activa participante en el proyecto de musealización.

Respecto a la valoración específica de las principales variables determinantes de la Fiesta de los Patios (tabla 6), las mujeres valoran en mayor medida los aspectos "Compra de souvenirs", "Ambiente de los patios", "Comodidad de la visita" y la "Distancia entre los patios en mayor medida " que los hombres $(p<0.05)$.

En todo caso, de las diferentes medias dimanadas de la tabla 6, debemos destacar que el aspecto más valorado por ambos grupos es la conservación de los patios (algo que es determinante de la calidad en la percepción de la visita), seguido por parte de los hombres por la integración en el entorno, y en el caso de las mujeres por el ambiente en los patios. Es necesario destacar también que una cuestión que siempre ha sido un aspecto de amplia polémica en la Fiesta de Patios, como es la espera en la visita, no es valorada negativamente por los encuestados; de hecho, los hombres le dan un valor de 3,46 puntos y las mujeres

Tabla 6. Valoración de diferentes aspectos de la Fiesta de los Patios. Comparación entre hombres y mujeres

\begin{tabular}{|l|c|c|c|c|c|c|}
\hline \multirow{2}{*}{ Aspectos } & \multicolumn{2}{|c|}{ Sexo } & \multicolumn{2}{c|}{ Prueba T para la igualdad de mediast } \\
\cline { 1 - 5 } & Hombre & Mujer & \multirow{2}{*}{ Dif. medias } & ET dif. & $\mathbf{t}_{881}$ & p \\
\cline { 2 - 6 } Conservación & $4,28(0,8)$ & $4,38(0,8)$ & $-0,10$ & 0,05 & $-1,87$ & 0,061 \\
\hline Tiempo de espera & $3,46(1,1)$ & $3,55(1,2)$ & $-0,09$ & 0,08 & $-1,13$ & 0,259 \\
\hline Señalización & $3,7(1,0)$ & $3,8(1,0)$ & $-0,10$ & 0,07 & $-1,45$ & 0,147 \\
\hline Información recibida & $3,68(1,0)$ & $3,81(1,0)$ & $-0,12$ & 0,07 & $-1,80$ & 0,073 \\
\hline Compra de souvenirs & $2,99(1,3)$ & $3,23(1,2)$ & $-0,24$ & 0,10 & $-2,44$ & $0,015^{*}$ \\
\hline Ambiente en los patios & $4,18(0,9)$ & $4,34(0,8)$ & $-0,16$ & 0,06 & $-2,79$ & $0,005^{* *}$ \\
\hline Comodidad de la visita & $3,95(0,9)$ & $4,09(0,9)$ & $-0,14$ & 0,06 & $-2,26$ & $0,024^{*}$ \\
\hline Accesibilidad a los Patios & $4,09(0,9)$ & $4,19(0,9)$ & $-0,10$ & 0,06 & $-1,70$ & 0,089 \\
\hline Integración en el entorno & $4,25(0,8)$ & $4,33(0,8)$ & $-0,09$ & 0,05 & $-1,59$ & 0,112 \\
\hline $\begin{array}{l}\text { Distancia entre los distintos } \\
\text { Patios }\end{array}$ & $3,87(1,0)$ & $4,01(1,0)$ & $-0,14$ & 0,07 & $-2,11$ & $0,035^{*}$ \\
\hline
\end{tabular}

† Homogeneidad asumida. ${ }^{*} \mathrm{p}<0.05 * * \mathrm{p}<0.01$ 
3,55 puntos. En el lado más bajo de las valoraciones $(3,23$ puntos para las mujeres, y no llegando si quiera al 3 para los hombres) y, por tanto, como aspecto de mejora más destacado, sestaría la posibilidad de realizar la compra de algún tipo de souvenirs relacionados con esta Fiesta, bien en los propios patios visitados o bien en establecimientos cercanos.

A continuación, se presentan en la tabla 7 los diferentes niveles de satisfacción en general con la Fiesta de los Patios, segmentándolos también entre hombres y mujeres.

De la tabla 7 podemos destacar que, el grado de satisfacción depende del sexo del entrevistado $\left[\chi_{2}^{2}=6.34\right.$; $p=0.042$ ]. El $88.4 \%$ de los hombres afirman estar satisfechos o muy satisfechos con la visita a los patios frente a un $92.5 \%$ de las mujeres, con lo que podemos afirmar que el porcentaje de mujeres satisfechas o muy satisfechas con la visita es mayor que el de hombres. En el resto de niveles de satisfacción no existen diferencias significativas.

Del mismo modo, en relación con la satisfacción de la Fiesta de los Patios, medida en una Escala de Likert de 5 puntos (1, muy poco satisfecho; 5 muy satisfecho), la satisfacción media global es bastante alta, alcanzando el 4,39. En la calificación dada por las mujeres se llega al 4,48, mientras que en el caso de los hombres es del 4,29. Por tanto con los datos desagregados, podemos afirmar que las mujeres están más satisfechas con la Fiesta de los Patios en general $(\mathrm{F}=15,660 ; p$-value $=0,000)$.

\section{CONCLUSIONES}

El estudio del patrimonio cultural inmaterial precisa de un enfoque multidisciplinar, donde geógrafos, etnólogos, sociólogos, antropólogos, historiadores y economistas incidan en aspectos de su investigación y protección. En este sentido, se ha descubierto la ne- cesidad imperiosa de salvaguardar dicho patrimonio, pues este nos provee de diversidad cultural, la cual nace de las raíces más profundas de la identidad de los pueblos, convertida en creatividad a través de las vivencias del pasado y transformándose en la memoria viva de nuestra forma de vida cultural y comunitaria. Pero además el patrimonio cultural inmaterial es un factor fundamental de desarrollo económico, principalmente a través del turismo que atrae, el cual, además, puede aportar la mejora en el conocimiento y en la difusión de las manifestaciones inmateriales de los pueblos.

El presente estudio trata de presentar los resultados de una de esas manifestaciones culturales intangibles, concretamente la Fiesta de los Patios de Córdoba, declarada Patrimonio Inmaterial de la Humanidad por la UNESCO en 2012, realizando un estudio de las características que definen esta tipología de patrimonio $\mathrm{y}$, a través del correspondiente trabajo de campo, y diferenciando por género, descubriendo el perfil sociodemográfico de los visitantes a la citada Fiesta y de sus motivaciones. De dicho estudio podemos concluir que, de manera general, el perfil sociodemográfico de los visitantes de la Fiesta de los Patios, es el de una persona con estudios universitarios, con una distribución en cuanto a la edad bastante similar, aunque prevalecen las personas con más de 60 años, con un nivel medio de renta y con una categoría profesional de asalariado. Dicho perfil establece los ámbitos poblacionales a los que deberían orientarse las diversas actuaciones de promoción y fomento que los entes públicos y privados que intervienen en el desarrollo turístico de la Fiesta de los Patios, realizan con el objetivo de incrementar el número de visitantes y su satisfacción. Pero también señala los nichos potenciales de mejora en este ámbito que permitan, entre otras cuestiones, rejuvenecer el público objetivo buscando la diversificación sociodemográfica.

Tabla 7. Niveles de satisfacción en general con la Fiesta de los Patios

\begin{tabular}{|l|c|c|c|}
\hline & \multicolumn{2}{|c|}{ Sexo $\mathbf{n}(\%)$} & \\
\hline Satisfacción & Hombre & Mujer & Total \\
\hline Muy poco satisfecho/ Poco satisfecho & $8 a(1.9)$ & $5 a(1.0)$ & $\mathbf{1 3}(\mathbf{1 . 4 )}$ \\
\hline Normal & $42 a(9.7)$ & $32 a(6.5)$ & $\mathbf{7 4}(\mathbf{8 . 0 )}$ \\
\hline Satisfecho/ Muy satisfecho & $382 a(88.4)$ & $459 b(92.5)$ & $\mathbf{8 4 1}(\mathbf{9 0 . 6 )}$ \\
\hline & $\mathbf{4 3 2 ( 1 0 0 . 0 )}$ & $\mathbf{4 9 6 ( 1 0 0 . 0 )}$ & $\mathbf{9 2 8 ( 1 0 0 . 0 )}$ \\
\hline
\end{tabular}

a,b: Cada letra de subíndice indica un subconjunto de sexo categorías cuyas proporciones de columna no difieren significativamente entre sí en el nivel ,05. 
También resalta la diversidad cultural de los extranjeros que visitan este patrimonio inmaterial, destacando en el trabajo de campo, la obtención de 39 diferentes nacionalidades. La asistencia de las entidades implicadas en la organización de la Fiesta de los Patios, a eventos y ferias internacionales especializadas, determinaría también una buena medida para su desarrollo turístico. En cuanto a la motivación principal para la visita a la ciudad, quizás por su mayor experiencia turística, destacan la visita al patrimonio cultural material que posee la ciudad de Córdoba, como esson el casco histórico (Judería) y la MezquitaCatedral. En este sentido, la convergencia del Patrimonio Material e Inmaterial de la Humanidad que posee la ciudad de Córdoba, haría necesario el desarrollo de estudios relacionados tanto con las ciencias de la conservación del patrimonio como con el desarrollo de programas de prácticas específicos en el ámbito universitario de Córdoba.
La principal limitación para la investigación se encuentra en la dificultad de la obtención de datos estadísticos oficiales diferenciados de la Fiesta de los Patios y de los otros eventos festivos que tiene la ciudad en el mismo mes, y, en general, de la visita a la propia ciudad.

Como futuras líneas de investigación se propone la realización de un análisis para profundizar en la relación y sinergias que pudieran existir entre el patrimonio material e inmaterial de la ciudad, buscando con ello conocer las posibilidades de asociación promocional de ambos y la forma en que la declaración de la UNESCO ha influido en su mayor conocimiento global y, simultáneamente, si esta cuestión hace que deba aumentarse la protección sobre los mismos. Adicionalmente, en las próximas ediciones de la Fiesta se recomienda realizar investigaciones de carácter cualitativo tanto entre los turistas como entre los visitantes de la propia comunidad local.

\section{BIBLIOGRAFÍA}

Aguilar Solves, M. P. (2014). Nuevos retos en la ciencia de la conservación del patrimonio. Revista ph, 85, pp. 18-19.

Alonso González, P. y Macías Vázquez, A. (2014). Bombardeando patrimonio en el Campo de Tiro Militar de El Teleno (León): de la sublimación de la naturaleza a la construcción social del patrimonio cultural. Arbor, 190 (766), a121. https://doi.org/10.3989/ arbor.2014.766n2013doi: http://dx.doi. org/10.3989/arbor.2014.766n2013

Ariño Villarroya, A. (2002). La expansión del patrimonio cultural. Revista de Occidente 250, pp. 129-150.

Arizpe, L. (2009). El patrimonio cultural inmaterial de México: ritos y festividades. México: Miguel Ángel Porrúa.

Bouchenaki, M. (2004). Editorial. Museum International. Intangible heritage, 56 (12), pp. 6-10.

Bouchenaki, M. y Kurin, R. (1989). Safeguarding Traditional Cultures: A Global Assessment of the 1989 UNESCO Recommendation on the Safeguarding of Traditional Culture and Folklore. Washington, D.C.: Center for Folklife and Cultural Heritage. Smithsonian Institution.

Boude Figueredo, O. y Luna, M. (2013). Gestión del conocimiento: salvaguardia del Patrimonio Cultural Inmaterial del Carnaval de Barranquilla. Opción, 29 (71), pp. 27-44.
Brisset, D. (1992). Rituales festivos de la provincia de Granada (actualidad y evolución histórica). II Las soldadescas. En: Anuario Etnológico de Andalucía 19881990. Sevilla: Consejería de Cultura y Medio Ambiente. Junta de Andalucía, pp. 183-191.

Brunet, I. y Alarcón, A. (2008). El turismo rural en Cataluña. Estrategias empresariales. Revista Internacional de Sociología, 66 (49), pp. 141-165.

Bruwer, J. (2004). The love affair of Generation-X consumers with the winery cellar door. The Australian \& New Zealand Grapegrower \& Winemaker 491, pp. 19-24.

Carrera Díaz, G. y Delgado Méndez, A. (2012). La antropologización del patrimonio y la patrimonialización de la cultura. Documentar el patrimonio etnológico en el IAPH. Revista Andaluza de Antropología, 2, pp. 108-127.

Castelli González, A. (2006). Museos de la ciudad, patrimonio cultural e identidad. BIRA 33, pp. 281-286. Disponible en http://revistas.pucp.edu.pe/index.php/ boletinira/article/viewFile/1974/1906

Cejudo, R. (2014). Sobre el valor del Patrimonio Cultural Inmaterial: una propuesta desde la ética del consumo. Dilemata, 14, pp. 189-209.

Chen, C. F., y Chen, F. S. (2010). Experience quality, perceived value, satisfaction and behavioural intentions for heritage tourists. Tourism Management, 31 (1), pp. 29-35. https://doi.org/10.1016/j. tourman.2009.02.008

Cuasapás Ponce, A. C. (2013). Estudio del valor turístico del patrimonio tangible e intangible del cantón Montúfar. [Trabajo de Grado inédito]. Ibarra, Ecuador

Desantes Real, M. (2013). Hacia una visión holística del patrimonio cultural. RIIPAC. Revista sobre Patrimonio Cultural, 3 (13), pp. 1-22.

Delgado Méndez, A. (2008). Barro, madera, cuero y oro: breve acercamiento al mundo de las artesanías en la ciudad de Écija. En Martín Pradas, A. (coord.) Actas de las VI Jornadas de Protección del Patrimonio Histórico de Écija. Patrimonio inmaterial de Écija II, costumbres, artesanía y tradiciones culinarias, Écija, del 25 al 27 de octubre de 2007. Écija: Asociación de Amigos de Écija, pp. 55-76.

Di Giovine, M. A. (2009). The Heritage-scape: UNESCO, World Heritage, and Tourism. Lexington Books.

Dodd, T. H. y Gustafson, A. W. (1997). Product, environmental, and service attributes that influence consumer attitudes and purchases at wineries. Journal of Food Products Marketing, 4 (3), pp. 41-59. https://doi.org/10.1300/ J038v04n03_04 
Fernández-Huerga, E. (2010). La teoría de la segmentación del mercado de trabajo: enfoques, situación actual y perspectivas de futuro. Investigación Económica, LXIX (273), pp. 115-150.

Finn, M., Walton, M. y Elliott-White, M. (2000). Tourism and Leisure Research Methods: Data Collection, Analysis and Interpretation. Harlow: Pearson Education.

García-Olivares, A. (1997). Tensión en el sistema de metáforas epistemológicas de la cultura contemporánea. Arbor, 158 (621), pp. 25-45. https://doi. org/10.3989/arbor.1997.i621.1810doi: 10.3989/arbor.1997.i621.1810

Gilabert González, L. M. (2015). Museos, gestión y patrimonio cultural: El proyecto de la ciudad de Oporto. PASOS. Revista de Turismo y Patrimonio Cultural, 13 (1), pp. 93-112. https://doi. org/10.25145/j.pasos.2015.13.007

Giménez, G. (2007). Estudios sobre la cultura y las identidades sociales. Guadalajara, México: Consejo Nacional para la Cultura y las Artes / Instituto Coahuilense de Cultura.

Gómez Pellón, E. (2002). El sentido práctico de la antropología social en el ámbito del patrimonio cultural. En Actas del VI Congreso de la Sociedad Española de Antropología Aplicada. Simposio: El sentido práctico de la antropología. Noviembre, 14, 15 y 16 de 2002, pp. 297-315. [En línea]. Disponible en https://es.scribd.com/ document/88743433/Seaa-El-SentidoPractico-De-La-Antropologia

Hernando Gonzalo, A. (2009). El patrimonio: entre la memoria y la identidad de la Modernidad. Revista ph, 70, pp. 88-97.

Howley, M. y Van Westering, J. (2000). Wine tourism in the United Kingdom. En Hall, C. M., Sharples, L., Cambourne, B. y Macionis, N. (eds.) Wine tourism around the world: Development, management and markets. Oxford: Butterworth-Heinemann, pp. 175-189.

Instituto Nacional de Estadística (2014a). Cifras oficiales de población resultantes de la revisión del Padrón municipal a 1 de enero de 2013. Madrid: Servicio de Publicaciones.

Instituto Nacional de Estadística (2014b). Encuesta de Ocupación Hotelera. Madrid: Servicio de Publicaciones.

Jolley, A. (2002). The wine industry, wine tourism and tourism in general. Documento de trabajo. Centre for Strategic Economic Studies of Victoria University, Melbourne

Kirshenblatt-Gimblett, B. (2004). El patrimonio inmaterial como producción metacultural. Museum International. Intangible Heritage, 221-222, pp. 52-67.

Kotler, P., Armstrong, G. Saunders, J. y Wong, V. (1999). Principles of Marketing. Upper Sadle River: Prentice Hall Inc.

Leijonhufvud, A. (2011). La naturaleza de una economía. Investigación Económica, 70 (277), pp. 15-36.

López López, J. D. (2011). Tradición, conflicto y valores en la fiesta: el patrimonio inmaterial desde un enfoque intercultural. En: García Castaño, F. J. y Kressova, N. (coords.). Actas del I Congreso Internacional sobre Migraciones en Andalucía. Granada: Universidad de Granada, Instituto de Migraciones, pp. 1893-1901.

López López, J. D. y Ruiz Ruiz, J. F. (2013). Identificación/producción del patrimonio inmaterial en Andalucía. Un análisis a través del caso de los Mosqueteros del Santísimo Sacramento de Béznar (Granada). Gazeta de Antropología, 29 (1), artículo 06.

Lourenço-Gomes, L., Costa Pinto, L. M. y Rebelo, J. F. (2014). Visitors' preferences for preserving the attributes of a World Heritage Site. Journal of Cultural Heritage, 15 (1), pp. 64-67. https://doi. org/10.1016/j.culher.2013.01.003

Mantecón, A. (1998). El patrimonio cultural. Estudios contemporáneos. Presentación. Alteridades, 8 (16), pp. 3-9.

Mariano, M. (2011). Patrimonio intangible e identidad: representaciones bolivianas en Olavarría, provincia de Buenos Aires, Argentina. Intersecciones en Antropología, 12 (1), pp. 83-94.

Nicholas, L. y Thapa, B. (2010). Visitor perspectives on sustainable tourism development in the Pitons Management Area World Heritage Site, St. Lucia. Environment, Development and Sustainability, 12 (5), pp. 839-857. https://doi. org/10.1007/s10668-009-9227-y

Nunnally, J. C. y Bernstein, I. H. (1994). Psychometric theory. New York: McGraw-Hill.

Olivera, A. (2011). Patrimonio inmaterial, recurso turístico y espíritu de los territorios. Cuadernos de Turismo, 27, pp. 663-677.

Plasencia Martín, M. (2007). El silbo gomero. Tradición viva de la cultura canaria y Patrimonio de la Humanidad. PA-
SOS. Revista de Turismo y Patrimonio Cultural, 5 (1), pp. 31-43. https://doi. org/10.25145/j.pasos.2007.05.003

Prat i Carós, J. (1999). Folklore, cultura popular y patrimonio. Sobre viejas y nuevas pasiones identitarias. Arxius de Sociologia, 3, pp. 87-109.

Quintana, C. y Stagno, R. (2009). Patrimonio y turismo: la activación turística patrimonial de Purificación (Paysandú, Uruguay). PASOS. Revista de Turismo y Patrimonio Cultural, 7, (2), pp. 307-319.

Quintero Rivera, A. G. (2002). Salsa, identidad y globalización. Redefiniciones caribeñas a las geografías y al tiempo. Trans. Revista Transcultural de Música, 6.

Quiros Vicente, F. J. y Casas Pérez, M. C. (2011). Antecedentes y consideraciones para la conservación del patrimonio cultural en el siglo XXI. Tlatemoani. Revista Académica de Investigación, 8, pp. 1-11.

Ruggles, F. y Silverman, H. (2009). Intangible Heritage Embodied. Nueva York: Springer

Sánchez Sánchez, N. y Fernández Puente, A. C. (2014). Desajuste educacional y de competencias: efectos diferenciales sobre la satisfacción laboral. Un estudio aplicado al mercado de trabajo español. Estudios de Economía, 41 (2), pp. 261-281. https://doi. org/10.4067/S0718-52862014000200005

Sánchez Carretero, C. (2005). Sobre el patrimonio inmaterial de la humanidad y la lucha por visibilizar "lo africano" en la República Dominicana. En Sierra Rodríguez, X. C. y Pereiro Pérez, X. (coords.) Patrimonio cultural: politizaciones $y$ mercantilizaciones. Sevilla: Federación de Asociaciones de Antropología del Estado Español, pp. 147-163.

Santamarina Campos, B. (2013). Los mapas geopolíticos de la UNESCO: entre la distinción y la diferencia están las asimetrías. El éxito (exótico) del patrimonio inmaterial. Revista de Antropología Social, 22, pp. 263-286. https://doi. org/10.5209/rev_RASO.2013.v22.43191

Tassiopoulos, D., Nuntsu, N. y Haydam, N. (2004). Wine Tourists in South Africa: A Demographic and Psychographic Study. Journal of Wine Research, 15 (1), pp. 51-63. https://doi. org/10.1080/0957126042000300326

Troncoso, C. A. y Almirón, A. V. (2005). Turismo y patrimonio. Hacia una relectura de sus relaciones. Aportes y Transferencias, 9 (1), pp. 56-74. 
UNESCO (2001). Proclamation of Masterpieces of the Oral and Intangible Heritage of Humanity. Guide for the Presentation of Candidature Files. [En línea]. Disponible en http://unesdoc.unesco.org/ images/0012/001246/124628eo.pdf

UNESCO (2003). Convención para la Salvaguardia del Patrimonio Cultural Inmaterial. Ratificada en la sesión 32 de la Conferencia General de la Organización de las Naciones Unidas para la Educación, la Ciencia y la Cultura en París, del 29 de septiembre al $17 \mathrm{de}$ octubre del 2003. [En línea]. Disponible en http://unesdoc.unesco.org/ images/0013/001325/132540s.pdf

Velasco Maillo, H. (1990). El folklore y sus paradojas. Revista Española de Investigaciones Sociológicas, 49, pp. 123-144. https://doi.org/10.2307/40183432
Villaseñor Alonso, I y Zolla Márquez, E. (2012). Del patrimonio cultural inmaterial o la patrimonialización de la cultura. Cultura y Representaciones Sociales, 6 (12), pp. 75-101.

Wedel, M. y Kamakura, W. A. (2000). Market Segmentation. Conceptual and Methodological Foundations. Dordrecht: Kluwer Academic Publishers. https://doi.org/10.1007/978-1-46154651-1

\section{Otros recursos}

Aspectos culturales, sociales, festivos, económicos y de vida cotidiana asociados al patio tradicional cordobés. IESA. Instituto de Estudios Sociales Avanzados. Informes y Monografías E-0834. [En línea]. Disponible en http://www.iesa. csic.es/publicaciones/300120150.pdf
Estudio sobre la repercusión económica en la ciudad de Córdoba de la Fiesta de los Patios en mayo de 2013. F \& J Martín. Abogados. [En línea]. Disponible en http://www.fj-martin.com/Informes/ ResumenPatios-Mayo2013.pdf

Observatorio Turístico Córdoba. Informe 2013. [En línea]. Disponible en http://www.turismodecordoba.org/84/gdocumental/ 16_a27_c1/informe_anual2013.pdf

Turismo y patrimonio cultural inmaterial. Organización Mundial del Turismo. Ética y responsabilidad social. [En línea]. Disponible en http://ethics.unwto.org/es/ content/turismo-y-patrimonio-culturalinmaterial 